\title{
A Novel Online Adaptive Time Delay Identification Technique
}

\author{
Alper Bayrak ${ }^{\star}$ and Enver Tatlicioglu
}

\begin{abstract}
In this study, an online time delay identification technique is proposed. Different from the relevant literature, the time delay is considered as a nonlinear parameter and nonlinear parameter estimation techniques are adopted. The stability of the adaptive update law can be guaranteed via Lyapunov based arguments and numerical simulations are conducted to demonstrate the proof of concept.
\end{abstract}

\section{INTRODUCTION}

Time delay is a widely came across phenomenon in dynamical systems in a wide variety of disciplines such as chemistry, biology, communications, mechanics, control, and signal processing applications. In dynamical systems, time delay may have negative effects such as instability and/or reduction in performance. On the other hand, its accurate identification is crucial for several signal processing applications such as distance measurement and localization systems [1], [2], [3], [4], [5]. Due to its occurrence in several disciplines and to overcome its negative effects, a significant amount of research was devoted to time delay phenomenon, its effects on systems, and identification and control methods. A broad overview on time delay, its effects on systems and open problems may be found in [6].

Identification of time delay in systems is a per se research area and several techniques and algorithms are available. In [7], Tugnait proposed an adaptive frequency domain filter based on high-order statistics for a class of error-in-variable models and applied this method to time delay identification. Zhou and Frank [8] developed an approach based on a modified tracking filter for time delay identification for a class of nonlinear autoregressive processes with exogenous inputs. Diop et al. [9] utilized an on-line estimation scheme based on least squares algorithm to identify time delay while providing exponential stability. In [10], So presented an unbiased impulse response estimation approach for time delay identification between signals received at two spatially separated sensors. Zhang and $\mathrm{Li}$ [11] analyzed the timevarying communication delay and proposed a time delay identification method based on steepest descent algorithm. Wen et al. proposed a new symmetrical adaptive structure to solve the problem of time delay identification in noisy environment and developed a stochastic gradient algorithm to calculate the optimum solution [12]. Shaltaf presented a neuro-fuzzy technique for identification of time delay embedded within a received noisy and delayed replica of a known reference signal [13]. Sharma and Joshi utilized

* To whom all the correspondence should be addressed.

The authors are with the Department of Electrical \& Electronics Engineering, Izmir Institute of Technology, Urla, Izmir, 35430 Turkey. E-mails: [alperbayrak, envertatlicioglu] @iyte.edu.tr fractional Fourier transform for time delay identification [14]. In [5], Brennan et al. presented the equivalence between time and frequency domain methods when estimating time delay and identified the conditions under which both methods are identical and then applied these methods to determine the position of leak in water distribution pipes. Govindan et al. [15] showed that the coherence analysis is suitable for time delay identification of narrow band coherence signals for which the conventional methods can not be reliably applied and used the method to identify time delay between two simultaneously measured signals. In [16], Harva and Raychaudhury proposed a Bayesian approach for identifying time delay between signals that are irregularly sampled. Bhardwaj and Nath [17] proposed a maximum likelihood identifier for time delay associated with each path in a multipath acoustic channel. Bastard et al. presented a modified version of the estimation of signal parameters via rotational invariance techniques algorithm which takes both transmitted pulse shape and any noise into account, to identify time delay in backscattered radar signals [18].

Most of the relevant past research on time delay identification are limited to linear (or linearized) time invariant systems [19]. Even in the studies that considered nonlinear systems, time delays were assumed to be appearing as linear parameters [20]. The proposed approaches in the literature usually use least squares algorithm, gradient algorithm, correlation analysis, filter based techniques, or stochastic approximation techniques [8]. A good categorization and comparison of time delay identification methods may be found in [21]. These approaches are efficient mostly for linear systems and also for some nonlinear systems where time delay is assumed to be a linear parameter. But, in reality, time delay acts as a nonlinear parameter. There are not many notable results in the literature for nonlinear systems. Most of the proposed algorithms in the literature, i) are generally weak under stability analysis, ii) do not apply to general systems, iii) and/or require measurability of some signals that are usually unavailable. For example, in [9], Diop et al. used an identification approach which provides exponential stability. But the assumption that obstruct zero crossing of the derivative of signal restricts the area of usage and furthermore, as indicated in the paper, in implementation, ultimate convergence can be provided instead of exponential convergence. Review of the relevant literature highlights the fact that time delay should be considered as a linear parameter for general systems and should not be considered as a linear parameter and thus nonlinear parameter identification techniques should be proposed for time delay identification. In this study, a novel online adaptive time delay identification 
technique is presented to identify constant time delays for the systems of the form in (1). Unlike most of the relevant past studies in the literature, time delays are considered as nonlinear parameters. Since time delay is treated as a nonlinear parameter, it is not possible to use a standard adaptive estimator or a gradient based estimator. To overcome this, inspired by [22], a nonlinear parameter identification technique is adopted as a time delay identification method. According to the authors' best knowledge, nonlinear parameter identification techniques are, for the first time in the literature, considered as a time delay identification method. The proposed time delay identification algorithm is based on a min-max optimization algorithm. It is shown that the developed estimator identifies the unknown time delay upon satisfaction of a nonlinear persistent excitation condition and is robust to noise as demonstrated by the numerical simulation results. The main contributions of this paper are (i) time delay is considered as a nonlinear parameter affecting the system and nonlinear parameter identification techniques are utilized as the time delay identification method, (ii) the developed identifier is continuous, and (iii) the developed identifier provides identification of time delay within a desired precision that can be adjusted to be very small.

\section{Plant Model}

The general model considered in this paper is of the following form

$$
\begin{array}{r}
\left.q(\tau, \Pi)=\Psi_{1}(t)+\Psi_{1}\left(t-\tau_{1}\right)\right)+\ldots+\Psi_{1}\left(t-\tau_{n}\right)+ \\
\ldots+\Psi_{m}(t)+\Psi_{m}\left(t-\tau_{1}\right)+\ldots+\Psi_{m}\left(t-\tau_{n}\right)
\end{array}
$$

which can be rewritten as

$$
q(\tau, \Pi)=\sum_{i=1}^{m} \Psi_{i}(t)+\sum_{i=1}^{m} \sum_{j=1}^{n} \Psi_{i}\left(t-\tau_{j}\right)
$$

where $q(\cdot) \in \mathbb{R}$ is a measurable signal, $\Pi(\cdot)$ is a measurable function including known and measurable parameters, $\Psi_{i}(t) \in \mathbb{R}, i=1 \cdots m$, are arbitrary chosen functions and $\tau=\left[\tau_{1} \cdots \tau_{n}\right]^{T} \in \mathbb{R}^{n}$ where $\tau_{j}, j=1,2, . ., n$ denote time delays. We assume that the time delays are unknown and all the remaining parameters are known. The signals shown below may be given as examples for the model given in (1)

$$
\begin{gathered}
q(\tau, \Pi)=\sin \left(2 \pi f_{1} t\right)+\sin \left(2 \pi f_{1} t-\tau_{1}\right) \\
q(\tau, \Pi)=\sin \left(2 \pi f_{1} t-\tau_{1}\right)+\sin \left(2 \pi f_{2} t\right) \\
q(\tau, \Pi)=\sin \left(2 \pi f_{1} t-\tau_{1}\right)+\sin \left(2 \pi f_{2} t-\tau_{2}\right)
\end{gathered}
$$

where $f_{1}$ and $f_{2}$ are frequencies, $\tau_{1}$ and $\tau_{2}$ are time delays and $\Pi(t)=t$. The model in (1) satisfies the conditions given in Assumptions 1, 2, 3, and 4.

Assumption 1: It is assumed that $\tau$, the unknown time delay vector, is bounded and in a known hypercube $\Omega \subset \mathbb{R}^{n}$.

Assumption 2: It is assumed that the function $q(\cdot)$ is either concave or convex on a simplex ${ }^{1} \Omega_{s}$ in $\mathbb{R}^{n}$, also $\Omega_{s} \supset \Omega$.

\footnotetext{
${ }^{1} \mathrm{~A}$ simplex in $\mathbb{R}^{n}$ is a convex polyhedron with $n+1$ vertices.
}

Definition 1: A function $W$ is convex on $\Omega$ if it satisfies the following inequality

$W\left(\kappa \nu_{1}+(1-\kappa) \nu_{2}\right) \leq \kappa W\left(\nu_{1}\right)+(1-\kappa) W\left(\nu_{2}\right), \forall \nu_{1}, \nu_{2} \in \Omega$

and concave if it satisfies the following inequality

$W\left(\kappa \nu_{1}+(1-\kappa) \nu_{2}\right) \geq \kappa W\left(\nu_{1}\right)+(1-\kappa) W\left(\nu_{2}\right), \forall \nu_{1}, \nu_{2} \in \Omega$

where $0 \leq \kappa \leq 1$.

Assumption 3: It is assumed that the function $\Pi(t)$ is continuous function of its arguments, bounded, and Lipschitz in $t$ as follows

$$
\left\|\Pi\left(t_{1}\right)-\Pi\left(t_{2}\right)\right\| \leq L_{1}\left|t_{1}-t_{2}\right|, \quad \forall t_{1}, t_{2} \in \mathbb{R}^{+}
$$

where $L_{1} \in \mathbb{R}^{+}$is the Lipschitz constant.

Assumption 4: It is assumed that $q\left(\tau_{0}, \Pi\right)$ is Lipschitz with respect to its arguments as

$$
\left|q\left(\tau_{0}+\Delta \tau_{0}, \Pi+\Delta \Pi\right)-q\left(\tau_{0}, \Pi\right)\right| \leq L_{2}\left(\|\Delta \Pi\|+\left\|\Delta \tau_{0}\right\|\right)
$$

where $\Delta \Pi \triangleq \Pi\left(t_{1}\right)-\Pi\left(t_{2}\right), \Delta \tau_{0} \triangleq \tau_{0}\left(t_{1}\right)-\tau_{0}\left(t_{2}\right)$ and $L_{2} \in \mathbb{R}^{+}$is the Lipschitz constant.

Remark 1: Affine functions always satisfy convexity and concavity defined in Definition 1 [23]. Therefore all affine functions are both convex and concave. Conversely, it can be said that all functions which are both convex and concave are affine (also linear). In Assumption 2, $q(\cdot)$ is assumed to be either convex or concave. Thus, this assumption is equivalent to excluding affine functions.

\section{Delay Estimation}

The estimate form of (2) is defined as

$$
\hat{q}=\sum_{i=1}^{m} \Psi_{i}(t)+\sum_{i=1}^{m} \sum_{j=1}^{n} \Psi_{i}\left(t-\hat{\tau}_{j}\right)
$$

where $\hat{q}=q(\hat{\tau}, \Pi) \in \mathbb{R}$. An auxiliary filter signal, denoted by $q_{f}(t) \in \mathbb{R}$, is designed as follows

$$
\dot{q}_{f}=-\alpha q_{f}+q \quad, \quad q_{f}\left(t_{0}\right)=0
$$

where $\alpha \in \mathbb{R}$ is a positive constant. The estimate form of (11) is designed as

$$
\dot{\hat{q}}_{f}=-\alpha\left(\hat{q}_{f}-\varepsilon s a t(r)\right)+\hat{q}-a^{*} \operatorname{sat}(r)
$$

where $\hat{q}_{f}(t), \dot{\hat{q}}_{f}(t) \in \mathbb{R}$ are the estimates of $q_{f}(t)$ and $\dot{q}_{f}(t)$, respectively, $\varepsilon \in \mathbb{R}$ is the desired precision, $a^{*}(t) \in \mathbb{R}$ is the tuning function and $r(t) \in \mathbb{R}$ is defined as follows

$$
r \triangleq \frac{\tilde{q_{f}}}{\varepsilon}
$$

where $\tilde{q}_{f}(t) \in \mathbb{R}$ is an error signal defined as follows

$$
\tilde{q}_{f} \triangleq \hat{q}_{f}-q_{f} \text {. }
$$

In (12), sat $(r)$ is a saturation function and defined as

$$
\operatorname{sat}(r)=\left\{\begin{array}{ll}
1, & r \geqslant 1 \\
r, & |r|<1 \\
-1, & r \leqslant-1
\end{array} .\right.
$$


After taking the time derivative of (14), the below expression may be obtained

$$
\dot{\tilde{q}}_{f}=-\alpha \tilde{q}_{f \varepsilon}+\hat{q}-q-a^{*} \operatorname{sat}(r)
$$

where (11) and (12) were utilized and $\tilde{q}_{f \varepsilon}(t) \in \mathbb{R}$ is the tuning error defined as

$$
\tilde{q}_{f \varepsilon} \triangleq \tilde{q}_{f}-\varepsilon s a t(r) .
$$

Remark 2: It should be noted that from (17) and its time derivative, it is clear that

$$
\begin{array}{lll}
\tilde{q}_{f \varepsilon}=0 & \text { when }\left|\tilde{q}_{f}\right| \leq \varepsilon \\
\dot{\tilde{q}}_{f \varepsilon}=\dot{\tilde{q}}_{f} & & \text { when }\left|\tilde{q}_{f}\right|>\varepsilon
\end{array}
$$

where (13) was utilized.

This remark will later be utilized in the stability analysis. The update law is developed with a projection as follows

$$
\dot{\hat{\tau}}=\operatorname{Proj}\left\{-\Gamma \tilde{q}_{f \varepsilon} \phi^{*}\right\}
$$

where $\phi^{*}(t) \in \mathbb{R}^{n}$ is the sensitivity function, $\Gamma \in \mathbb{R}^{n \times n}$ is a positive definite diagonal gain matrix and the projection algorithm assures that $\hat{\tau}(t)$ always belongs to the hypercube $\Theta$. The projection algorithm is as

$$
\hat{\tau}_{j}= \begin{cases}\hat{\tau}_{j} & , \quad \text { if } \hat{\tau}_{j} \in\left[\tau_{j, \min }, \tau_{j, \text { max }}\right] \\ \tau_{j, \text { min }}, & \text { if } \hat{\tau}_{j}<\tau_{j, \text { min }} \\ \tau_{j, \text { max }}, & , \text { if } \hat{\tau}_{j}>\tau_{j, \text { max }}\end{cases}
$$

where the subscript $\mathrm{j}$ denotes the $j^{\text {th }}$ element of the corresponding vector $\forall j=1,2, \ldots n, \tau_{j, \min }, \tau_{j, \max } \in \mathbb{R}$ are the minimum and maximum values of the $j^{\text {th }}$ component of $\tau$, respectively. The solutions for $\phi^{*}(t)$ and $a^{*}(t)$ are obtained from the following min-max optimization problem ${ }^{2}$

$$
\begin{gathered}
a^{*}=\min _{\phi \in \mathbb{R}^{n}} \max _{\tau \in \Omega_{s}} J(\phi, \tau) \\
\phi^{*}=\arg \min _{\phi \in \mathbb{R}^{n}} \max _{\tau \in \Omega_{s}} J(\phi, \tau)
\end{gathered}
$$

where $J(\cdot) \in \mathbb{R}$ is performance index and given as follows

$$
J(\cdot)=\operatorname{sat}(r)\left[\hat{q}-q-\Gamma \tilde{\tau}^{T} \phi\right]
$$

where $\tilde{\tau}(t) \in \mathbb{R}^{n}$ is the parameter estimation error defined as follows

$$
\tilde{\tau} \triangleq \hat{\tau}-\tau .
$$

The expressions in (20) and (21) can be obtained as follows a) when $\tilde{q}_{f}(t)<0$

$$
\begin{gathered}
a^{*}= \begin{cases}0 & \text { if } q \text { is concave on } \Theta_{\mathrm{s}} \\
A_{1} & \text { if } q \text { is convex on } \Theta_{\mathrm{s}}\end{cases} \\
\phi^{*}= \begin{cases}\nabla q(\hat{\tau}) & \text { if } q \text { is concave on } \Theta_{\mathrm{s}} \\
A_{2} & \text { if } q \text { is convex on } \Theta_{\mathrm{s}}\end{cases}
\end{gathered}
$$

b) when $\tilde{q}_{f}(t) \geqslant 0$

$$
a^{*}= \begin{cases}A_{1} & \text { if } q \text { is concave on } \Theta_{\mathrm{s}} \\ 0 & \text { if } q \text { is convex on } \Theta_{\mathrm{s}}\end{cases}
$$

\footnotetext{
${ }^{2}$ Although the derivations are very similar to that of [23], we presented them for the sake of completeness.
}

$$
\phi^{*}= \begin{cases}A_{2} & \text { if } q \text { is concave on } \Theta_{\mathrm{s}} \\ \nabla q(\hat{\tau}) & \text { if } q \text { is convex on } \Theta_{\mathrm{s}}\end{cases}
$$

where $A(t) \in \mathbb{R}^{n+1}$ is given as follows

$$
A=\left[\begin{array}{ll}
A_{1} & A_{2}^{T}
\end{array}\right]^{T}=G^{-1} b
$$

where $A_{1}(t) \in \mathbb{R}, A_{2}(t) \in \mathbb{R}^{n}$ and $G(t) \in \mathbb{R}^{(n+1) \times(n+1)}$ and $b(t) \in \mathbb{R}^{n+1}$ are defined as

$$
\begin{gathered}
G=\left[\begin{array}{cc}
-1 & \beta \Gamma\left(\hat{\tau}-\tau_{s 1}\right)^{T} \\
-1 & \beta \Gamma\left(\hat{\tau}-\tau_{s 2}\right)^{T} \\
-1 & \beta \Gamma\left(\hat{\tau}-\tau_{s 3}\right)^{T} \\
-1 & \beta \Gamma\left(\hat{\tau}-\tau_{s 4}\right)^{T}
\end{array}\right] \\
b=\left[\begin{array}{c}
\beta\left(\hat{q}-q_{s 1}\right) \\
\beta\left(\hat{q}-q_{s 2}\right) \\
\beta\left(\hat{q}-q_{s 3}\right) \\
\beta\left(\hat{q}-q_{s 4}\right)
\end{array}\right]
\end{gathered}
$$

where $\beta \in \mathbb{R}$ is defined as follows

$$
\beta= \begin{cases}1 & \text { if } q \text { is convex on } \Theta_{\mathrm{s}} \\ -1 & \text { if } q \text { is concave on } \Theta_{\mathrm{s}} .\end{cases}
$$

In (30), $q_{s h} \triangleq q\left(\tau_{s h}, \Pi\right) \forall h=1,2, \ldots, n+1$ where $\tau_{s h} \in$ $\mathbb{R}^{n}$ are the vertices of the simplex $\Theta_{s}$. In (25) and (27), $\nabla q(\hat{\tau}) \in \mathbb{R}^{n}$ is the gradient function given as follows

$$
\nabla q(\hat{\tau})=\left.(\delta q / \delta \tau)\right|_{\tau=\hat{\tau}} .
$$

Remark 3: The hypercube $\Omega$ may be obtained by using minimum and maximum values of $\tau$. The vertices of the simplex $\Omega_{s}$ may be obtained by first inscribing $\Omega$ in a $n$ dimensional sphere and then inscribing this sphere inside a $(n+1)$-dimensional polyhedron [22].

Remark 4: The tuning error $\tilde{q}_{f \varepsilon}(t)$ and the saturation function $\operatorname{sat}(r)$ assure that the estimator is continuous even if a discontinuous solution of the min-max algorithm is obtained [22].

\section{Stability Analysis}

Theorem 1: The adaptive update law in (18) assures that $\tilde{q}_{f \varepsilon}(t) \in L_{2} \cap L_{\infty}$; hence, the stability of the identifier and the global boundedness of the overall adaptive system are guaranteed. The estimator assures that $\|\tilde{\tau}(t)\| \leqslant \sqrt{\gamma}$ as $t \rightarrow \infty$ provided the following nonlinear persistent excitation condition holds

$$
\beta\left(\Pi\left(t_{2}\right)\right)\left(q\left(\hat{\tau}\left(t_{1}\right), \Pi\left(t_{2}\right)\right)-q\left(\tau, \Pi\left(t_{2}\right)\right)\right) \geqslant \varepsilon_{u}\left\|\hat{\tau}\left(t_{1}\right)-\tau\right\|
$$

where $\gamma, c_{1} \in \mathbb{R}$ are positive constants defined as

$$
\gamma \triangleq \frac{8 \varepsilon c_{1}}{\varepsilon_{u}^{2}}, \quad c_{1} \triangleq 4 L_{1} L_{2}+2 \nu L_{2} L_{\phi}+\nu L_{\phi}^{2}
$$

where $\nu \in \mathbb{R}$ is the maximum eigenvalue of $\Gamma, t_{2} \in$ $\left[t_{1}, t_{1}+T_{0}\right], t_{1}>t_{0}$, and $T_{0}, \varepsilon_{u} \in \mathbb{R}$ are positive constants. Proof: Although the systems considered in this work are completely different from the one in [23], and the update law in (18) is slightly different than the one in [23] (i.e. unlike our work, there is no gain matrix in the adaptive update rule (see equation (31)) in [23]), the proof of the theorem is similar to the one in [23]. In this paper, the stability analysis is given 
in appendix and the reader is referred to [23] for the rest of the proof.

Remark 5: From its definition in (34), it is clear that $\gamma$ can be made smaller by choosing a smaller precision $\varepsilon$. It's also clear that as $\varepsilon \rightarrow 0$ then $\gamma \rightarrow 0$, thus the time delay identification error also goes to zero in the sense that $\|\tilde{\tau}(t)\| \rightarrow 0$.

\section{Numerical Simulation Results}

The performance of the proposed technique was evaluated by conducting numerical simulation using Matlab/Simulink. During the simulation, the update law in (18) was utilized with gains and parameters $\alpha=250, \Gamma=150$ and $\varepsilon=$ 0.0001 . The lower and upper bounds of time delay were 0.1 seconds and 1.1 seconds, respectively. The initial values of $q_{f}(t)$ and $\hat{q}_{f}(t)$ were set to 0 and the initial value of $\hat{\tau}(t)$ was 0.3 seconds.

The model in (3) was considered with $f_{1}=5 \mathrm{~Hz}$ and $\tau_{1}=$ 0.6 seconds. The performance of the proposed technique was evaluated with and without additive noise where white Gaussian noise with a $20 \mathrm{~dB}$ SNR was injected to $q(t)$ to demonstrate robustness against noise. The estimate of $\tau_{1}$ is presented in Figures 1 and 2, for noiseless and noisy cases, respectively. From Figures, it is clear that identification of time delay is achieved even in the presence of noise.

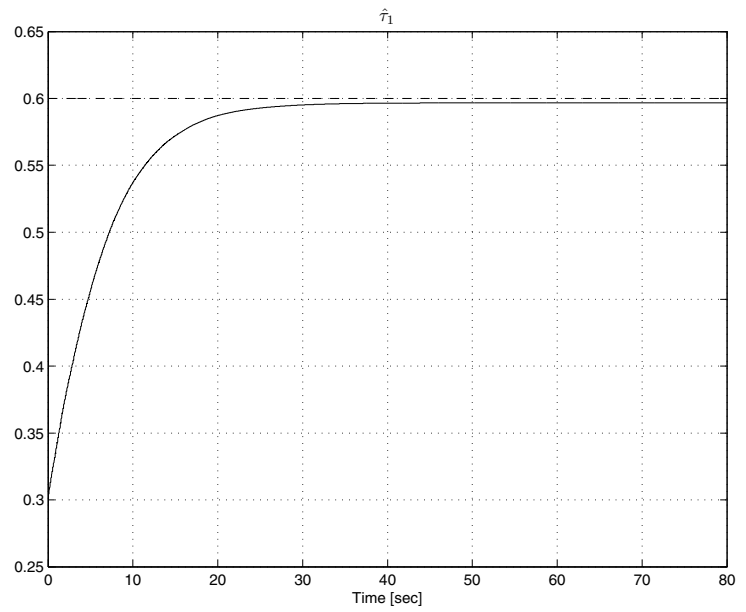

Fig. 1. $\hat{\tau}_{1}(t)$ for constant delay without noise

\section{CONCLUSION}

In this work, a novel adaptive time delay identification technique was presented. Time delays were considered as nonlinear parameters and the nonlinear parameter estimation method [22] was utilized as the time delay identification algorithm for the first time in the literature. Numerical simulation results were conducted to demonstrate the efficiency of the estimator for constant time delays and its robustness to noise. From the results, it is clear that the developed technique efficiently identifies constant time delays. In future, this technique will be adapted to general

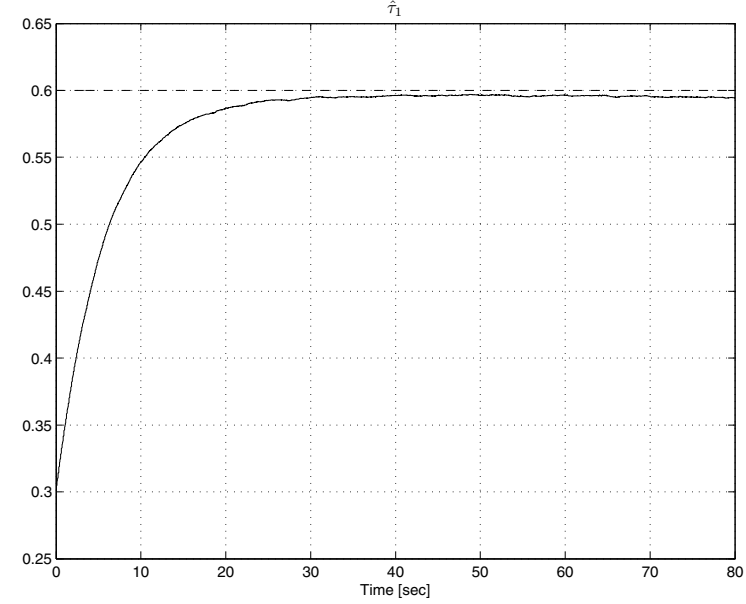

Fig. 2. $\hat{\tau}_{1}(t)$ for constant delay with noise

classes of systems with control applications.

\section{APPENDIX}

Proof: To facilitate the proof, a nonnegative Lyapunov function $V(t) \in \mathbb{R}$ is defined as follows

$$
V=\frac{1}{2} \tilde{q}_{f \varepsilon}^{2}+\frac{1}{2} \tilde{\tau}^{T} \tilde{\tau}
$$

The time derivative of (35) can be obtained as follows

$$
\dot{V}=\tilde{q}_{f \varepsilon} \dot{\tilde{q}}_{f \varepsilon}+\tilde{\tau}^{T} \dot{\tilde{\tau}}
$$

After utilizing the time derivative of (23), the expression given in (36) can be written as follows

$$
\dot{V}=\tilde{q}_{f \varepsilon} \dot{\tilde{q}}_{f \varepsilon}+\tilde{\tau}^{T} \dot{\hat{\tau}} .
$$

After substituting (18) into (37), the following expression is obtained

$$
\dot{V}=\tilde{q}_{f \varepsilon} \dot{\tilde{q}}_{f \varepsilon}+\tilde{\tau}^{T} \operatorname{Proj}\left\{-\Gamma \tilde{q}_{f \varepsilon} \phi^{*}\right\} .
$$

It should be noted that an adaptive law with the projection algorithm defined on a convex set retains all the properties of the adaptive law without the projection algorithm [24]. The projection strategy given in (19) is on the hypercube $\Theta$ (i.e., a convex set); hence, the expression given in (38) can be written as follows

$$
\dot{V}=\tilde{q}_{f \varepsilon} \dot{\tilde{q}}_{f \varepsilon}-\tilde{\tau}^{T} \Gamma \tilde{q}_{f \varepsilon} \phi^{*} .
$$

The expression given in (39) is rearranged as follows

$$
\dot{V}=\tilde{q}_{f \varepsilon}\left[\dot{\tilde{q}}_{f \varepsilon}-\Gamma \tilde{\tau}^{T} \phi^{*}\right] .
$$

Two different cases are considered, Case I when $\left|\tilde{q}_{f}\right| \leq \varepsilon$, and Case II when $\left|\tilde{q}_{f}\right|>\varepsilon$.

Case I) From Remark 2, it follows that

$$
\dot{V}=0 \quad \forall\left|\tilde{q}_{f}\right| \leq \varepsilon .
$$


Case II) Also, from Remark 2 and (40), the following expression can be obtained

$$
\dot{V}=\tilde{q}_{f \varepsilon}\left[\dot{\tilde{q}}_{f}-\Gamma \tilde{\tau}^{T} \phi^{*}\right] \quad \forall\left|\tilde{q}_{f}\right|>\varepsilon .
$$

After substituting (16) into (42), the following expression is obtained

$$
\dot{V}=\tilde{q}_{f \varepsilon}\left[-\alpha \tilde{q}_{f \varepsilon}+\hat{q}-q-a^{*} \operatorname{sat}(r)-\Gamma \tilde{\tau}^{T} \phi^{*}\right] .
$$

The expression given in (43) can be rearranged as follows

$$
\dot{V}=-\alpha \tilde{q}_{f \varepsilon}^{2}+\tilde{q}_{f \varepsilon}\left[\hat{q}-q-\Gamma \tilde{\tau}^{T} \phi^{*}-a^{*} \operatorname{sat}(r)\right] .
$$

Now two distinct sub-cases of Case II are considered: (a) when $\tilde{q}_{f}>\varepsilon$, and (b) when $\tilde{q}_{f}<-\varepsilon$. (a) When $\tilde{q}_{f}>\varepsilon$, from (15) and (17) it follows that $\tilde{q}_{f \varepsilon}>0$ and $\operatorname{sat}(r)=$ $\operatorname{sgn}\left(\tilde{q}_{f}\right)=1$. After utilizing (44), $\dot{V}(t)$ can be written as follows

$$
\dot{V}=-\alpha \tilde{q}_{f \varepsilon}^{2}+\tilde{q}_{f \varepsilon}\left[\hat{q}-q-\Gamma \tilde{\tau}^{T} \phi^{*}-a^{*}\right] .
$$

It follows from (45) that $\dot{V}(t) \leq 0$ if the following inequality holds

$$
a^{*} \geq \hat{q}-q-\Gamma \tilde{\tau}^{T} \phi^{*} \quad \forall \tau \in \Theta .
$$

Therefore, we choose to maximize $a^{*}(t)$ as follows

$$
a^{*}=\max _{\tau \in \Theta}\left[\hat{q}-q-\Gamma \tilde{\tau}^{T} \phi^{*}\right] \quad \text { for any } \phi^{*} .
$$

Since, $a^{*}(t)$ is like a gain in (16), we seek to find $\phi^{*}(t)$ so that $a^{*}(t)$ is minimized; thus, $a^{*}(t)$ is chosen as follows

$$
a^{*}=\min _{\phi \in \mathbb{R}^{n}} \max _{\tau \in \Theta}\left[\hat{q}-q-\Gamma \tilde{\tau}^{T} \phi^{*}\right] .
$$

(b) When $\tilde{q}_{f}<-\varepsilon$, from (15) and (17) it follows that $\tilde{q}_{f \varepsilon}<$ 0 and $\operatorname{sat}(r)=\operatorname{sgn}\left(\tilde{q}_{f}\right)=-1$. After utilizing $(44), \dot{V}(t)$ can be written as follows

$$
\dot{V}=-\alpha \tilde{q}_{f \varepsilon}^{2}+\tilde{q}_{f \varepsilon}\left[\hat{q}-q-\Gamma \tilde{\tau}^{T} \phi^{*}+a^{*}\right] .
$$

From (49) it follows that $\dot{V}(t) \leq 0$ if the following inequality holds

$$
a^{*} \geq q-\hat{q}+\Gamma \tilde{\tau}^{T} \phi^{*} \quad \forall \tau \in \Theta .
$$

Following along the same lines as in (a), the following expression can be written

$$
a^{*}=\min _{\phi \in \mathbb{R}^{n}} \max _{\tau \in \Theta}\left[q-\hat{q}+\Gamma \tilde{\tau}^{T} \phi^{*}\right] .
$$

After combining (48) and (51), the following expression is obtained

$$
a^{*}=\min _{\phi \in \mathbb{R}^{n}} \max _{\tau \in \Theta} \operatorname{sat}(r)\left[\hat{q}-q-\Gamma \tilde{\tau}^{T} \phi^{*}\right] .
$$

After utilizing (46) and (50) the following inequality can be obtained

$$
\operatorname{sat}(r)\left[\hat{q}-q-\Gamma \tilde{\tau}^{T} \phi^{*}\right]-a^{*} \leq 0 .
$$

The expression given in (45) can be rewritten as follows

$$
\dot{V}=-\alpha \tilde{q}_{f \varepsilon}^{2}+\tilde{q}_{f \varepsilon} \operatorname{sat}(r)\left\{\operatorname{sat}(r)\left[\hat{q}-q-\Gamma \tilde{\tau}^{T} \phi^{*}\right]-a^{*}\right\}
$$

Thus, after utilizing (53), and the fact that $\tilde{q}_{f \varepsilon} \operatorname{sat}(r) \geq 0$ when $\left|\tilde{q}_{f}\right|>\varepsilon, \dot{V}(t)$ can be upper bounded as follows

$$
\dot{V} \leq-\alpha \tilde{q}_{f \varepsilon}^{2} \quad \forall\left|\tilde{q}_{f}\right|>\varepsilon .
$$

After integrating (55), the following inequality can be obtained

$$
\alpha \int_{t_{0}}^{\infty} \tilde{q}_{f \varepsilon}^{2} d \tau<V\left(t_{0}\right)-V(\infty) .
$$

From (35), (41), and (55), it can be concluded that $V(t) \in$ $\mathcal{L}_{\infty}$. From (56), it is clear that $\tilde{q}_{f \varepsilon}(t) \in \mathcal{L}_{2} \cap \mathcal{L}_{\infty}$; thus, from (17), it can be concluded that $\tilde{q}_{f}(t) \in \mathcal{L}_{\infty}$. Since the projection strategy given in (19) ensures $\hat{\tau}(t) \in \Theta_{s}$; thus, it follows that $\hat{\tau}(t) \in \mathcal{L}_{\infty}$. Hence, from (12), it follows that $\hat{q}(\cdot) \in \mathcal{L}_{\infty}$. Since $a^{*}(t)$ is a function of the bounded signals, and $q(\cdot)$ is a measurable bounded signal, from (16), it follows that $\dot{\tilde{q}}_{f}(t) \in \mathcal{L}_{\infty}$. It is clear from the projection strategy that $\dot{\hat{\tau}}(t) \in \mathcal{L}_{\infty} ;$ thus, from $(23), \dot{\tilde{\tau}}(t) \in \mathcal{L}_{\infty}$.

\section{REFERENCES}

[1] P. Giraudet and H. Glotin, "Real-time 3d tracking of whales by echorobust precise tdoa estimates with a widely-spaced hydrophone array," Applied Acoustics, vol. 67, no. 11-12, pp. 1106 - 1117, 2006.

[2] J. M. Martin, A. R. Jimenez, F. Seco, L. Calderon, J. L. Pons, and R. Ceres, "Estimating the 3d-position from time delay data of uswaves: experimental analysis and a new processing algorithm," Sensors and Actuators A: Physical, vol. 101, no. 3, pp. 311 - 321, 2002.

[3] K. W. Lui, F. K. Chan, and H. So, "Accurate time delay estimation based passive localization," Signal Processing, vol. 89, no. 9, pp. 1835 $-1838,2009$

[4] Y. Gao, M. Brennan, and P. F. Joseph, "On the effects of reflections on time delay estimation for leak detection in buried plastic water pipes," Journal of Sound and Vibration, vol. 325, no. 3, pp. 649 - 663, 2009.

[5] M. Brennan, Y. Gao, and P. Joseph, "On the relationship between time and frequency domain methods in time delay estimation for leak detection in water distribution pipes," Journal of Sound and Vibration, vol. 304, no. 1-2, pp. $213-223,2007$.

[6] J.-P. Richard, "Time-delay systems: an overview of some recent advances and open problems," Automatica, vol. 39, no. 10, pp. 16671694,2003

[7] J. K. Tugnait, "Frequency domain adaptive filters using higher-order statistics with application to adaptive time delay estimation," International Journal of Adaptive Control and Signal Processing, vol. 10, no. 2-3, pp. 137 - 157, 1996.

[8] D. H. Zhou and P. M. Frank, "A real-time estimation approach to timevarying time delay and parameters of narx processes," Computers \& Chemical Engineering, vol. 23, no. 11-12, pp. 1763 - 1772, 2000.

[9] S. Diop, I. Kolmanovsky, P. E. Moraal, and M. van Nieuwstadt, "Preserving stability/performance when facing an unknown timedelay," Control Engineering Practice, vol. 9, no. 12, pp. 1319 - 1325 , 2001.

[10] H. C. So, "Noisy input-output system identification approach for time delay estimation," Signal Processing, vol. 82, no. 10, pp. 1471 - 1475, 2002.

[11] T. Zhang and Y. Li, "A control scheme for bilateral teleoperation systems based on time-varying communication delay identification," in Proc. of Systems and Control in Aerospace and Astronautics, Harbin, China, 2006, pp. $273-278$

[12] J. Wen, P. Li, and Y. Wen, "A new method for unbiased time-delay estimation in noisy environments," International Journal of Adaptive Control and Signal Processing, vol. 21, no. 7, pp. 623 - 634, 2007.

[13] S. Shaltaf, "Neuro-fuzzy based time-delay estimation using dct coefficients," ISA Transactions, vol. 46, no. 1, pp. 21-30, 2007.

[14] K. K. Sharma and S. D. Joshi, "Time delay estimation using fractional fourier transform," Signal Processing, vol. 87, no. 5, pp. 853 - 865, 2007.

[15] R. Govindan, J. Raethjen, F. Kopper, J. Claussen, and G. Deuschl, "Estimation of time delay by coherence analysis," Physica A: Statistical Mechanics and its Applications, vol. 350, no. 2-4, pp. 277 - 295, 2005 
[16] M. Harva and S. Raychaudhury, "Bayesian estimation of time delays between unevenly sampled signals," Neurocomputing, vol. 72, no. 1-3, pp. $32-38,2008$.

[17] T. P. Bhardwaj and R. Nath, "Maximum likelihood estimation of time delays in multipath acoustic channel," Signal Processing, vol. 90, no. 5, pp. $1750-1754,2010$.

[18] C. L. Bastard, V. Baltazart, and Y. Wang, "Modified esprit (m-esprit) algorithm for time delay estimation in both any noise and any radar pulse context by a gpr radar," Signal Processing, vol. 90, no. 1, pp. $173-179,2010$.

[19] S. Drakunov, W. Perruquetti, J.-P. Richard, and L. Belkoura, "Delay identification in time-delay systems using variable structure observers," Annual Reviews in Control, vol. 30, no. 2, pp. 143-158, 2006.

[20] L. Belkoura and J.-P. Richard, "A distribution framework for the fast identification of linear systems with delays," in Proc. of 6th IFAC workshop on time delay systems, L'Aquila, Italy, 2006.

[21] S. Bjorklund and L. Ljung, "A review of time-delay estimation techniques," in Proc. of 42nd IEEE Conference on Decision and Control, vol. 3, Maui, HA, USA, 2003, pp. $2502-2507$.

[22] A. M. Annaswamy, F. P. Skantze, and A.-P. Loh, "Adaptive control of continuous time systems with convex/concave parametrization," Automatica, vol. 34, no. 1, pp. 33 - 49, 1998.

[23] N. Nath, E. Tatlicioglu, and D. M. Dawson, "Range identification for nonlinear parameterizable paracatadioptric systems," Automatica, vol. 46, no. 7, pp. 1129 - 1140, 2010.

[24] P. Ioannou and J. Sun, Robust Adaptive Control. Englewood Cliffs, NJ, USA: Prentice-Hall, 1996. 